la revue La revue pour l'histoire du CNRS

POUR L'HISTOIRE DU CNRS

$22 \mid 2008$

La géopolitique mode d'emploi

\title{
Le tas de sable Du désordre qui rassemble
}

Daniel Bideau, Étienne Guyon et Jeanne Pullino

\section{(2) OpenEdition}

Journals

Édition électronique

URL : https://journals.openedition.org/histoire-cnrs/8272

DOI : 10.4000/histoire-cnrs.8272

ISSN : 1955-2408

Éditeur

CNRS Éditions

Édition imprimée

Date de publication : 3 octobre 2008

ISBN : 978-2-271-06676-3

ISSN : 1298-9800

\section{Référence électronique}

Daniel Bideau, Étienne Guyon et Jeanne Pullino, «Le tas de sable Du désordre qui rassemble », La revue pour l'histoire du CNRS [En ligne], 22 | 2008, mis en ligne le 03 octobre 2010, consulté le 20 mai 2021. URL : http://journals.openedition.org/histoire-cnrs/8272 ; DOI : https://doi.org/10.4000/histoirecnrs.8272

Ce document a été généré automatiquement le 20 mai 2021.

Comité pour l'histoire du CNRS 


\title{
Le tas de sable Du désordre qui rassemble
}

\author{
Daniel Bideau, Étienne Guyon et Jeanne Pullino
}

Une communauté scientifique entre originalité et excellence

1 À travers l'histoire des Miam, c'est toute une aventure scientifique qui nous est contée. Cette action, toujours aussi vivace actuellement par son fonctionnement informel, implique des acteurs provenant de laboratoires français du CNRS et de quelques partenaires européens. Elle n'est pas la seule, heureusement, mais l'évolution des thèmes et le renouvellement continu des acteurs tout en conservant la permanence de la structure, en font une structure modèle. L'attachement aux rencontres annuelles de Carry le Rouet, véritable fil conducteur historique ${ }^{1}$, et l'intérêt non démenti pour les Miam, maillon central de la science de la matière en désordre à l'interface entre plusieurs départements du CNRS (physique, chimie, mécanique, géosciences) sont deux invariants.

2 Pendant toutes ces années, les tutelles ont joué un rôle d'incitation et d'accompagnement indispensable, en particulier le CNRS qui a soutenu une structure très peu standard avec intelligence, souplesse et avec une aide financière assez limitée. Cette communauté est l'heureux résultat d'une complicité scientifique amicale au sein d'un petit groupe de chercheurs avec, comme figure emblématique, Pierre-Gilles de Gennes².

1960-1980 : la vie avant les Miam

3 À la fin des années 1960, à coté de la physique des solides cristallins qui a connu de grands succès dont découle le développement actuel des technologies électroniques, une physique des matériaux désordonnés plus mécanique et s'appuyant sur la physique statistique se fait sentir.

De la physique des solides à la matière mal condensée

4 Après la seconde guerre mondiale, la physique des solides est bien représentée au CNRS grâce à des personnalités telles que Louis Néel, Pierre Aigrain, André Guinier, Jacques Friedel et Raymond Castaing, ces trois derniers ayant créé un institut CNRS de physique des solides à l'université d'Orsay (le LA2) reconnu au niveau mondial. Pierre-Gilles de 
Gennes rejoindra ce laboratoire en 1960. Les avancées ${ }^{3}$, en particulier en physique électronique (semi-conducteurs, supraconducteurs et magnétisme), ont été fulgurantes. Dans les années 1970, cette discipline s'est employée à produire, à caractériser et à étudier des matériaux solides possédant des états en volume et surface très propres et contrôlés, recourant à des expérimentations sophistiquées (ultravide, caractérisation in situ). Cette période est aussi marquée, a contrario, par une transition vers des matériaux désordonnés et vers des matériaux moins classiques tels que les cristaux liquides et les polymères.

5 L'école de physique théorique des Houches, dont la création après-guerre a contribué au redémarrage de la physique en France, est le témoin de cette évolution. En 1978, l'école porte le titre de Physique mal condensée ; elle est suivie deux ans plus tard par une seconde école, Physique des défauts. On parle alors de matériaux sales ${ }^{4}$. La première de ces écoles se consacrera essentiellement au désordre au niveau microscopique (défauts, verres, amorphes, alliages). Le cours de Philip Anderson ${ }^{5}$ sur l'étude de la localisation y fait date. Cette théorie, qui ne sera comprise que très progressivement, montre qu'un désordre, aussi faible soit-il, change le comportement qualitatif d'un matériau par rapport à un état sans désordre. Dans la seconde école en 1980, Étienne Guyon introduit le terme Miam dans un cours intitulé Macroscopic Random Media ou MIlieux Aléatoires Macroscopiques où il décrit une ouverture des concepts microscopiques bien connus de la physique statistique des systèmes désordonnés (liquides, gaz, verres) à la physique des milieux poreux, des suspensions et des gels.

6 Une réflexion prospective sur les matériaux a fait l'objet de plusieurs rapports. Un premier rapport conclut un programme d'action concertée sur les matériaux que préside Jacques Friedel. Il s'inscrit dans la ligne d'une action continue en métallurgie et la création d'un premier DEA sur les matériaux. L'action Matériaux (à la DGRST) qui lui fait suite en 1973 conduira à un rapport de Jean-Pierre Causse, alors directeur scientifique de Saint-Gobain, qui est un plaidoyer en faveur des études des matériaux à propriétés finalisées et de l'interaction entre science de base et génie des matériaux.

Ce rapport sera remis finalement au ministre de l'Éducation nationale, Jean-Pierre Chevènement, en 1981. Cette réflexion conduira à la création du Programme interdisciplinaire de recherches sur les matériaux (Pirmat) en mars 1982, résultant d'un transfert de moyens des actions concertées vers le CNRS. Un rapport, demandé par la direction de la chimie du CNRS, par Michel Cournil sur Phases divisées, phases dispersées sera utilisé pour la première demande de soutien de l'activité Miam faite par P.-G. de Gennes et É. Guyon au CNRS en 1979.

Une première activité de recherche à Paris, Marseille et Rennes

Des recherches partant du thème de la percolation et des applications de la mécanique des fluides (rhéologie des gels, milieux poreux, composites électriques) vont d'abord se développer dans un laboratoire créé par É. Guyon en 1977 à l'ESPCI. É. Guyon, qui avait accepté une mission de restructuration de la physique à l'université de Provence ${ }^{6}, \mathrm{y}$ lance des recherches sur les mélanges granulaires. L'association d'un important laboratoire parisien et d'un ensemble d'une demi-douzaine de chercheurs plutôt isolés et à $700 \mathrm{~km}$ de distance semblait peu raisonnable! Et pourtant, elle a parfaitement fonctionné conduisant un peu plus tard à la création de l'URA 1000, la plus étendue de France! Robert Chabbal, alors directeur général du CNRS, avait créé un poste de secrétaire pour l'accompagnement de cette mission, affecté, à partir de 1980, à Jeanne Pullino. Une petite équipe rennaise dirigée par Daniel Bideau rejoindra bientôt les deux 
structures partenaires. Ces trois équipes mettront en place une structure de collaboration nationale centrée à Marseille et autour de rencontres annuelles à Carry, toujours active aujourd'hui.

Les rencontres annuelles de Carry

9 À la différence des instituts permanents aux Houches, à Aussois ou Cargèse, les rencontres annuelles de Carry sont, depuis 30 ans, organisées dans un VVF en bord de mer près de Marseille, ce qui a permis une organisation administrative légère avec un coût assez modeste de ce type d'établissement, et des conditions d'accueil assez spartiates les premières années. Plusieurs composantes à ce succès : la continuité d'une organisation administrative à Marseille (aujourd'hui dans le cadre de l'unité associée IUSTI), la priorité donnée à des conférences plus ou moins formelles de scientifiques reconnus français et étrangers, et la souplesse de cette gestion scientifique.

Le professeur Nicolas Rivier parle de " a program decided on the first morning in the car ride between Saint-Charles and Carry». Et pourtant, cela a fonctionné! Des collaborations durables s'y sont mises en place avec des ouvertures disciplinaires: le génie des procédés avec Jacques Villermaux (Nancy), la mécanique des fluides avec John Hinch (Cambridge) ou la physique statistique avec Dietrich Stauffer (Cologne). Cette présence internationale s'est poursuivie jusqu'à nos jours: on peut noter une présence américaine (Jim Jenkins et Michel Louge, de Cornell University, Tony Rosato du New Jersey Institute of Technology, P. Lacour Gayet, J. Willemsen et P. N. Sen Schlumberger Ridgefield, Sidney Nagel de Chicago), israélienne (Halm Tsohar et Hezi Yizhaq, université de Ben-Gourion)...

11 Ces rencontres de Carry présentent un profil sociologique atypique: une nette décentralisation des laboratoires ( $60 \%$ de provinciaux) ; un subtil mélange des genres et des disciplines; une population plus jeune que la moyenne; un processus de renouveau permanent dû à des thèmes porteurs et à un large échange intellectuel. Un regret peut-être : la faible présence industrielle (10\% environ de l'effectif) malgré une volonté affichée d'ouverture vers le monde industriel (LCPC, EDF, Saint- Gobain, RhônePoulenc, IFP, Lafarge...).

Les années 1980 : les actions thématiques programmées

12 La décennie suivante verra, avec la création d'une RCP, un nombre important d'études de modélisation utilisant les outils et les modèles de la physique statistique des systèmes microscopiques transposés à des systèmes de désordre à plus grande échelle. L'action Miam a été soutenue pour la première fois par un programme d'aides relevant de l'objectif «science des matériaux ». Le premier comité d'ATP était présidé par P.-G. de Gennes avec une participation équilibrée d'industriels, de métallurgistes, céramistes, et encore peu de physiciens des Miam. Cette action était couplée à une autre action portant sur le frittage des poudres métalliques et céramiques, mettant en jeu des matériaux désordonnés mais plus finalisée. L'action « céramiques » se justifiait par les espoirs d'applications telles que les moteurs de voitures ou le projet spatial Hermès. La fragilité des pièces réalisées n'a pas permis de concrétiser cette ambition. Les ingénieurs des matériaux de construction, les géophysiciens et même les militaires se sont eux aussi intéressés aux travaux sur les matériaux hétérogènes.

13 Le changement politique de 1981 a accéléré l'affichage par Programmes interdisciplinaires de recherche (PIR) avec un soutien direct du nouveau directeur général Pierre Papon, favorable aux actions inter départements. Celui-ci créera d'ailleurs une dizaine de PIR, dont le Pirmat en 1982 en réponse aux besoins 
économique et industriel de nouveaux matériaux. Un premier PIR, le Pirdes ${ }^{7}$, avait été créé autour de l'énergie solaire un peu plus tôt (1976) par Robert Chabbal, directeur général du CNRS. Jean Hanus, membre du comité initial puis directeur du Pirmat à partir de 1982, pilotera l'action avec ténacité, brio et ouverture scientifique, s'appuyant sur des personnalités éminentes de la physique telles que Jacques Friedel, Jacques Winter, directeur de la physique au CNRS, ou de certains industriels.

En 1982, 11 programmes d'ATP fonctionnent avec une trentaine d'actions retenues sur 300 projets déposés. L'action Miam reçoit, pour sa part, $750 \mathrm{kF}$ et retient 5 projets sur 27 présentés. Le dénominateur commun de ces projets : l'ouverture disciplinaire mêlant génie des procédés, physicochimie des matériaux désordonnés, mécanique des matériaux, physique du désordre, modélisation... On y retrouve les concepts de percolation, de fractales, de localisation, mettant en avant les effets spécifiques du désordre et contrastant avec les approches plus traditionnelles, visant à une homogénéisation du désordre (l'homogénéisation mathématique évoque un tableau pointilliste de Seurat où on s'intéresserait aux effets moyens de couleur), des mécaniciens et des spécialistes du génie des procédés.

La physique des solides et la physique électronique présentes en début du programme (supraconducteurs, composites électriques) auront tendance à disparaître, tandis que les applications en mécanique des fluides et des solides prendront une grande importance.

Le programme d'ATP est redéfini en 1988 sous l'appellation matériaux hétérogènes. Le passage de milieux à matériaux indique une volonté de rapprochement de systèmes réels plus que de systèmes modèles ainsi que la prise en compte d'effets macroscopiques plus proches de la tradition mécanique. Par ailleurs, les appels d'offres indiquent une préférence pour l'étude des propriétés non linéaires et de la réponse irréversible des matériaux à de fortes sollicitations mécaniques (endommagement, rupture, vieillissement...), hydrodynamiques (le bouchage d'un filtre par exemple), ou électriques. Elle marque aussi une ouverture progressive vers un dialogue avec la géophysique et les sciences de la Terre.

17 La programmation autour du Pirmat s'éteindra progressivement à la fin des années 1980. Les directions des départements (tout comme le directeur général Serge Feneuille, pourtant spécialiste des matériaux) rechignent à poursuivre cet effort parce qu'il échappe à leur contrôle et à la mainmise de leur département scientifique. Jean Hanus et son associée Marie-Claude Vitorge partiront en 1990 pour rejoindre le CEA avec pour objectif le lancement d'un programme similaire qui ne verra pas le jour! Malgré toutes les difficultés, l'action Miam se prolongera naturellement, grâce à la continuité des rencontres de Carry et d'un style scientifique qui perdure encore aujourd'hui.

Les années 1990 : un Groupe de recherche (GDR) sur les milieux hétérogènes complexes

Les années 1990 sont marquées par la création d'un groupe de recherche (GDR). Le soutien du ministère de la Recherche lui accordera une autonomie à la fois pour des rencontres et pour des financements de recherche. Alors que les années pionnières étaient souvent marquées par des expériences simples que justifiait le défrichage du sujet (on a parlé de «bricolos " à propos de l'activité d'une des équipes), l'on voit apparaître des instruments plus sophistiqués ainsi qu'un recours plus poussé à l'ordinateur. L'un des thèmes prioritaires sera celui de l'étude des milieux poreux et des milieux granulaires. Si, en 1980, on recensait peu de publications sur ces sujets dans les 
journaux internationaux de physique, elles seront courantes $^{8}$ dans ces mêmes journaux à partir de 1990 .

19 Le département des sciences physiques et mathématiques (SPM) propose d'accueillir l'action des Miam dans une sorte de laboratoire sans mur regroupant un certain nombre d'équipes de recherche et de laboratoires. Cette formation, Physique des milieux hétérogènes complexes (PMHC), voit le jour le 1er janvier 1991. Dans sa version initiale, il regroupe 21 laboratoires allant de la physique théorique à la mécanique des sols en passant par la géologie. Il est soutenu par deux départements du CNRS : SPM et SPI. Les directeurs en sont Daniel Bideau (université de Rennes I) et Stéphane Roux (ESPCI). La dotation de fonctionnement accordée par le CNRS durant chacune des quatre premières années sera en moyenne de $180000 \mathrm{~F}$. Les thèmes fondateurs initiaux de ce GDR sont au nombre de quatre: les milieux granulaires secs en écoulement; les suspensions de particules; les milieux poreux; les ondes dans les matériaux désordonnés... avec, en toile de fond, l'apport essentiel des techniques d'acquisition et de traitement d'images couplées à des traitements numériques rendus possibles par les nouvelles générations d'ordinateurs et d'acquisition d'images.

20 La première année de sa création, le GDR PMHC a eu la chance de recevoir un soutien spécifique substantiel $(800000 \mathrm{~F})$ du ministère de la Recherche qui lui permettra de lancer de nouvelles activités et d'entreprendre des collaborations avec un appel d'offres interne au GDR. Ce GDR a alors connu un grand succès. Alors que la durée normale d'un GDR est de 4 ans, celui-ci sera renouvelé pour 4 ans. Les actions aussi se sont centrées sur le thème des milieux granulaires secs et humides. Le GDR a aussi joué un rôle actif dans la formation par la recherche en facilitant et en organisant des échanges de doctorants entre les différentes équipes le composant avec des stages courts de un ou plusieurs mois. Ces échanges seront facilités et internationalisés par l'obtention d'un réseau Capital humain et mobilité de l'UE associant l'Angleterre, l'Allemagne, la Norvège et les Pays-Bas.

21 L'action a bénéficié de la présence de plusieurs scientifiques étrangers ${ }^{9}$ qui ont animé régulièrement les rencontres de Carry et ont été associés à des travaux de recherches. John Hinch, professeur au Trinity College et chercheur au DAMTP de Cambridge, imprégné de ce pragmatisme très britannique et d'une culture incontestable en mécanique, a accompagné en 1993 le lancement d'une activité de conseil scientifique collégial à l'échelle du GDR. Celle-ci consiste en une semaine de travail bloqué avec une entreprise, le but étant d'analyser et de proposer des solutions à des problèmes industriels posés par un groupe industriel. La première rencontre eut lieu avec le groupe Lafarge- Coppée. Ce premier essai, s'étant révélé positif, a été poursuivi avec Saint-Gobain, les Charbonnages de France (Agglocentre) et le LCPC.

2000 à 2008 : le GDR MIlieux DIvisés (MIDI)

Aujourd'hui, la communauté ne cesse de s'élargir, ce qui nécessite le travail en sousgroupes d'études. Les rencontres de Carry restent la clef de voûte de vie commune dans cette aventure scientifique. Un nouveau GDR, MIlieux DIvisés (MIDI) ${ }^{10}$, renouvelé une fois en 2004, a été créé en 2000. Si l'étude des milieux granulaires reste prioritaire, le GDR s'ouvre davantage à la géophysique, la géotechnique, la science des sols et vers les applications, tout en conservant sa part d'études sur des systèmes modèles.

23 Nouveaux intervenants, nouvelle équipe pilote: Bruno Andreotti à Paris, Olivier Pouliquen à Marseille, Frank Radjai à Montpellier... Les outils utilisés par cette 
communauté resteront les mêmes que précédemment mais s'enrichiront de nouvelles actions ${ }^{11}$. Les Miam n'ont pas fini de faire parler d'eux. À suivre donc ${ }^{12} \ldots$

Les Miam, et après?

En l'espace de 30 ans, la communauté Miam a peu à peu trouvé sa place et consolidé ses points forts :

- un ensemble d'acquis scientifiques dans des thématiques variées telles que la dynamique des dunes et avalanches, l'hydrogéologie et la récupération assistée, les applications des gels, la mécanique des composites...

- un renouvellement permanent de la communauté de chercheurs, véritable moteur de cette dynamique ;

- une bonne gestion de l'interdisciplinarité ;

- le bien-fondé du choix de la thématique avec une orientation à caractère fondamental, l'utilisation de nouveaux outils (modélisation numérique et imagerie) et l'apparition de nouveaux questionnements;

- une forte implication en termes de communication et de formation;

- un soutien institutionnel évident ;

- une grande souplesse organisationnelle.

Tout au long de ces années, cette communauté a reposé sur les épaules d'un groupe d'amis d'âges et de séniorités variés, en totale confiance dans le suivi d'un projet commun.

\section{Remerciements}

Nos remerciements vont d'abord à Robert Blanc qui a accompagné avec intelligence et souplesse, à l'université de Provence, cette action pendant plus de 20 ans. Nous associons à ces remerciements tous les chercheurs qui ont fait vivre cette communauté jusqu'à ce jour, en particulier, Stéphane Roux et Olivier Pouliquen qui nous ont aussi aidés à la préparation de ce document. Nous avons eu d'utiles échanges sur cet article avec, Bernadette Bensaude-Vincent, Jean Hanus, Pierre Papon et Marie-Claude Vitorge. Nous avons bénéficié d'une lecture critique de Jacques Friedel dont l'action autour de la physique des solides et des matériaux a été constante depuis les années 1950. Enfin, un clin d'oeil amical à Madame Dastrevigne, directrice de l'Espace Vacanciel, qui a fait preuve de beaucoup de souplesse et de compréhension pendant ces 30 années.

\section{NOTES}

1. Nous disposons aussi d'archives relativement complètes de la vie de cette communauté qui sont gérées dans le laboratoire CNRS de IUSTI à Marseille qui en assure le secrétariat (http://iusti.polytech.univmrs. fr/Miam).

2. Bien qu'ayant laissé la place aux plus jeunes, Pierre-Gilles de Gennes était encore présent aux rencontres de Carry de 1998 et 2000.

3. Le prix Nobel attribué en 2007 à Albert Fert, chercheur dans ce laboratoire dès cette époque, rappelle que cette recherche reste féconde aujourd'hui. 
4. Si ces qualificatifs apparaissent quelque peu dépréciatifs, les organisateurs de la première École rappellent que Galilée parlait de taches (maculae) solaires que nous acceptons tous aujourd'hui !

5. Philip Warren Anderson, prix Nobel de physique en 1977.

6. Mission que lui avait confiée le CNRS (via son directeur, Robert Chabbal) à la suite de la séparation entre cette université (U1) et une nouvelle université (U3) où se retrouvaient en majorité les seniors.

7. Voir Guthleben D. «Coup de soleil au CNRS... La genèse du Pirdes ». In La revue pour l'histoire du CNRS, n 17 , été 2007, pp. 36-39. L'article évoque cette création qui fit suite à la crise du pétrole.

8. Nul n'a été surpris de voir un château de sable sur la couverture de Nature de juin 1997 où était traité le problème de la stabilité du sable mouillé !

9. De nombreuses écoles thématiques et congrès ont été organisés : mini colloques aux Journées de la matière condensée de la société française de physique à Lille (1992) et Rennes (1994), école Otan à Cargèse « Mobile Particulate Systems » (1994), école de Porquerolles « Milieux granulaires secs et humides » en 1995 auxquelles s'ajoutent une école Otan à Cargèse « Physics of Dry Granular Media » en septembre 1997, une journée thématique « Milieux Divisés » au département SPM du CNRS et des ateliers sur la formation et la migration des dunes à en Tunisie en avril 1999, puis en Mauritanie deux ans plus tard, autour d'une thématique lancée par le GDR.

10. Un article de revue on dense granular flow portant la signature du GDR MIDI publié en 2004 dans l'European physical journal E., fait un point sur les activités issues de la communauté des Miam.

11. Les informations concernant cette dernière phase de l'activité Miam sont bien décrites dans l'important rapport d'activité de septembre 2007 que l'on retrouve sur le site des Miam. On y trouve aussi un ensemble de propositions concernant l'avenir de cette communauté. On y note la préoccupation des responsables qui voient les difficultés de devoir gérer un groupe trop gros et qui, du coup, ne pourra maintenir la complicité scientifique et la convivialité qui ont été des moteurs de cette action.

12. Un nouveau GDR « Mécanique et physique multiéchelles » (MePhy) vient d'être créé. Il doit renforcer les échanges entre physique et mécanique.

\section{RÉSUMÉS}

Un tas de sable, une gelée de groseilles, un composite conducteur, un filtre poreux... Derrière ces exemples concrets du quotidien se cache une science complexe, dédiée aux milieux aléatoires macroscopiques, les Miam. Depuis plus de trente ans, des générations de chercheurs ont collaboré dans le cadre d'une structure légère soutenue par le CNRS pour percer les mystères de la matière en désordre.

A pile of sand, a jelly, a conducting composite, a porous filter... Behind these various examples of everyday life is hidden a complex science dedicated to macroscopic random media (Miam, for MIlieux Aléatoires Macroscopiques). During the last thirty years, generations of scientists have collaborated in a flexible association to unravel the mysteries of random matter. 


\section{AUTEURS}

\section{DANIEL BIDEAU}

Daniel Bideau est professeur émérite, membre de l'institut de physique de Rennes (université de Rennes I, Équipe « Granulaires-Mousses »).

\section{ÉTIENNE GUYON}

Étienne Guyon est professeur émérite et travaille au Laboratoire de physique et mécanique des milieux hétérogènes de l'École supérieure de physique et de chimie industrielles de la ville de Paris.

\section{JEANNE PULLINO}

Jeanne Pullino est ingénieur d'études au CNRS à l'Institut universitaire des systèmes thermiques industriels (IUSTI, université de Provence). 